\title{
Recurrent evolution of gut symbiotic bacteria in pentatomid stinkbugs
}

Takahiro Hosokawa ${ }^{1,2+}$, Yu Matsuura ${ }^{1,3+}$, Yoshitomo Kikuchi ${ }^{4}$ and Takema Fukatsu ${ }^{1,5,6^{*}}$ (D)

\begin{abstract}
Background: Diverse animals are intimately associated with microbial symbionts. How such host-symbiont associations have evolved is a fundamental biological issue. Recent studies have revealed a variety of evolutionary relationships, such as obligatory, facultative, and free-living, of gut bacterial symbiosis within the stinkbug family Pentatomidae, although the whole evolutionary picture remains elusive.

Results: Here we investigated a comprehensive assembly of Japanese pentatomid stinkbugs representing 28 genera, 35 species, and 143 populations. Polymerase chain reaction (PCR), cloning, and sequencing of bacterial $16 \mathrm{~S}$ rRNA gene from their midgut symbiotic organ consistently detected a single bacterial species from each of the insect samples, indicating a general tendency toward monosymbiotic gut association. Bacterial sequences detected from different populations of the same species were completely or nearly identical, indicating that the majority of the gut symbiotic associations are stably maintained at the species level. Furthermore, bacterial sequences detected from different species in the same genus tended to form well-supported clades, suggesting that host-symbiont associations are often stable even at the genus level. Meanwhile, when we compared such sequences with published sequences available in DNA databases, we found a number of counter-examples to such stable host-symbiont relationships; i.e., symbionts from different host species in the same genus may be phylogenetically distant, and symbionts from the same host species may be phylogenetically diverse. Likewise, symbionts of diverse pentatomid species may be closely related to symbionts of other stinkbug families, and symbionts of diverse pentatomid species may even be allied to free-living bacteria. Molecular evolutionary analyses revealed that higher molecular evolutionary rates, higher AT nucleotide compositions, and smaller genome sizes tended to be associated with the pentatomid symbionts constituting the stable lineages, whereas these traits were rarely observed in the pentatomid symbionts of promiscuous type.

Conclusions: These results indicate that gut symbiotic bacteria have evolved repeatedly and dynamically in the stinkbug family Pentatomidae, which have plausibly entailed frequent symbiont acquisitions, losses, replacements and transfers, while establishing a number of relatively stable host-symbiont associations. The diverse host-symbiont relationships observed in the Pentatomidae will provide an ideal arena for investigating the evolution of symbiosis experimentally and theoretically.
\end{abstract}

Keywords: Stinkbug, Pentatomidae, Gut symbiont, y-Proteobacteria, $16 \mathrm{~S}$ rRNA gene, Molecular evolution, Reductive genome evolution

\footnotetext{
* Correspondence: t-fukatsu@aist.go.jp

${ }^{\dagger}$ Equal contributors

${ }^{1}$ National Institute of Advanced Industrial Science and Technology (AIST),

Tsukuba 305-8566, Japan

${ }^{5}$ Department of Biological Sciences, Graduate School of Science, University of

Tokyo, Tokyo 113-0033, Japan

Full list of author information is available at the end of the article
} 


\section{Background}

Many insects that rely on nutritionally limited dietary resources, such as plant sap, vertebrate blood, and woody material, are obligatorily associated with beneficial symbiotic microorganisms harbored within the gut lumen, body cavity, or cells. Without these microbial associates, the host insects are often unable to grow, survive, or reproduce normally. Conversely, symbiotic microorganisms are often unable to proliferate outside the host body, presumably because of adaptation to the intra-host environment. In this way, the host insect and the microbe are integrated into an almost inseparable biological entity, constituting an intimate symbiotic system of obligate nature [1-3]. Originally, however, such highly specialized symbiotic bacteria must have been derived from lessspecialized free-living bacteria. The origins and mechanisms underlying the obligate host-symbiont associations are of fundamental ecological and evolutionary interest.

The majority of plant-sucking stinkbugs (Insecta: Hemiptera: Pentatomoidea) possess a symbiotic organ in a posterior region of the midgut, in which numerous crypts develop and harbor a dense population of specific symbiotic bacteria [1, 4-7]. In these stinkbugs, reproducing females excrete symbiont-containing materials from the anus to the eggs or newborns, which are exploited by the offspring to establish vertical symbiont transmission. When experimentally deprived of symbiotic bacteria, host stinkbugs suffer substantial fitness defects, including retarded growth, elevated mortality, morphological abnormalities, reduced offspring, and/or complete sterility, indicating the biological importance of this symbiosis [1, 8-24].

Despite the general importance of symbiotic bacteria for host stinkbugs, evolutionary patterns of the hostsymbiont relationships differ markedly in different stinkbug lineages. In the families Plataspidae, Acanthosomatidae and Urostylididae, for example, the symbiont phylogeny mirrors the host phylogeny and the symbiont genomes are drastically reduced to less than $1 \mathrm{Mb}$, indicating stable host-symbiont co-speciation and degenerative genome evolution based on strict vertical symbiont transmission over evolutionary time $[11,12,18]$. In the families Pentatomidae and Cydnidae, by contrast, the symbiont phylogeny is not concordant with the host phylogeny, and the symbiont genomes exhibit no or moderate size reduction, suggesting relatively younger host-symbiont associations through acquisitions, horizontal transfers, and/or replacements of the symbiotic bacteria [13, 17, 20, 22, 23, 25]. Recent studies have revealed the striking diversity and dynamic evolutionary trajectories of gut symbiotic bacteria in the stinkbug family Pentatomidae: all gut symbiotic bacteria belong to the Enterobacteriaceae of the $\gamma$-Proteobacteria [10, 1315, 20-24, 26-28]; however, the symbiotic bacteria are polyphyletic and their phylogeny does not reflect the phylogeny of their host stinkbugs, suggesting multiple evolutionary origins of the symbiotic bacteria from Pantoea spp. and allied $\gamma$-proteobacteria $[13,20,22$, 23, 27, 28]; some symbionts are uncultivable whereas others can be cultivated outside the host body [22]. In some cases, multiple obligate symbiotic bacteria may coexist in the same host species, different stinkbug species may share the same symbiont species, and some environmental bacteria can establish stable infection and mutualistic association with these stinkbug species [22]. A more comprehensive picture of the evolutionary dynamics of the symbiotic bacteria in the Pentatomidae is thus desirable.

In this study, we surveyed the gut symbiotic bacteria of a comprehensive assemblage of pentatomid stinkbugs representing 28 genera, 35 species, and 143 populations in Japan, by which their diversity, phylogenetic relationship, and molecular and genomic evolution were investigated.

\section{Materials \\ Insect samples}

The stinkbug samples examined in this study are listed in Additional file 1. These insects were collected in Japan mostly as adults, and either taken to the laboratory alive or preserved in acetone [29].

\section{DNA analyses}

Fresh insects were individually dissected in phosphate

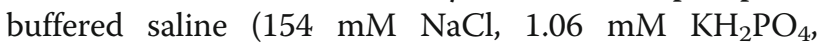
$2.97 \mathrm{mM} \mathrm{Na}_{2} \mathrm{HPO}_{4}$ ) with fine forceps under a dissection microscope. Acetone-preserved insects were dissected in the same way in $70 \%$ ethanol. Each dissected symbiotic organ of the midgut fourth section with numerous symbiont-harboring crypts was subjected to DNA extraction using QIAamp DNA Mini kit (Qiagen). A $1.5 \mathrm{~kb}$ segment of bacterial 16S rRNA gene was amplified by PCR using primers 16SA1 (5'-AGA GTT TGA TCM TGG CTC AG-3') and 16SB1 (5'-TAC GGY TAC CTT GTT ACG ACT T-3'), and cloned and sequenced as described [30]. The nucleotide sequences determined in this study were deposited in the DNA Data Bank of Japan (http://www.ddbj.nig.ac.jp/index-e.html) under accession numbers LC168475-LC168617 (see Additional file 1).

\section{Molecular phylogenetic and evolutionary analyses}

Multiple alignments of the nucleotide sequences were constructed by the program MAFFT v7.271 (G-INS-i) [31] and gap-containing regions and ambiguous sites of the alignment were removed manually. The nucleotide substitution model, GTR + I + G, was selected using the program jModeltest 2 based on the Akaike information criterion [32, 33]. The phylogenetic analyses were 
conducted by Bayesian and maximum-likelihood (ML) methods using the programs MrBayes v3.2.6 [34] and RAxML v8.1.5 [35], respectively. In the Bayesian analysis, multiple independent runs with four simultaneous Markov chains were performed for 50,000,000 generations, producing 50,001 trees (sample freq $=1,000$ ) in each run. After discarding the first 12,501 samples as 'burn-in', a total of 75,000 trees were used to generate majority rule consensus trees and calculate the posterior probabilities. In the ML analysis, the best-scoring ML tree was searched and the supported values were calculated by 1000 rapid bootstraps. Calculations of genetic distances and relative rate tests were performed by the program RRTree [36].

\section{Results and discussion}

\section{$16 \mathrm{~S}$ rRNA gene sequences of gut symbiotic bacteria of} pentatomid stinkbugs

From the midgut symbiotic organs of all 143 stinkbug individuals, representing 28 genera and 35 species, bacterial $16 \mathrm{~S}$ rRNA gene was subjected to PCR amplification, cloning and sequencing. For each sample, five clones were sequenced and all yielded the same sequence, indicating monosymbiotic status of pentatomid stinkbugs in general. Within the same species, the sequences were either completely identical (most of the species) or nearly identical (ex. Alcimocoris japonensis $>99.86 \%$; Dybowskyia reticulate $>99.86 \%$; Glaucias subpunctatus $>99.86 \%$; Gonopsis affinis >99.93\%; Graphosoma rubrolineatum >99.93\%) (Additional file 1). Within the same genus, sequences from different species tended to show high similarities to each other (ex. Homalogonia spp. 98.64\%; Menida spp. 93.89-98.10\%; Pentatoma spp. 98.29\%; Scotinophara spp. 97.69-98.23\%).

\section{Molecular phylogenetic analysis of gut symbiotic bacteria of pentatomid stinkbugs}

In addition to the 16S rRNA gene sequences of the gut symbiotic bacteria of the pentatomid stinkbugs determined in this study, we retrieved already-published $16 \mathrm{~S}$ rRNA gene sequences of gut symbiotic bacteria of pentatomid stinkbugs [13-15, 20-22, 26, 28] (Additional file 2), those of gut symbiotic bacteria of other stinkbugs representing the families Scutelleridae, Cydnidae, Parastrachiidae, Acanthosomatidae, Plataspidae and Urostylididae [15, 18, 25, 37-41] (Additional file 2), and those of closely-related free-living bacteria [42-52] (Additional file 2). Molecular phylogenetic analysis revealed that the gut symbionts of the pentatomid stinkbugs were all placed within the Enterobacteriaceae of the $\gamma$-Proteobacteria (Fig. 1). Of these, the gut symbionts of Scotinophara spp. constituted a distinct basal clade, clustering with the gut symbionts of Edessa spp. and the genome-reduced gut symbiont, Candidatus Benitsuchiphilus tojoi, of the stinkbug family Parastrachiidae. The other stinkbug gut symbionts formed a large and coherent cluster together with such free-living $\gamma$-proteobacteria as Pantoea, Enterobacter, Erwinia, etc. Among them, the gut symbionts of Nezara spp. clustered with the genome-reduced gut symbionts of other stinkbug families, Candidatus Ishikawaella capsulata of the Plataspidae, Candidatus Rosenkranzia clausaccus of the Acanthosomatidae, and Candidatus Tachikawaea gelatinosa of the Urostylididae. Outside of this cluster were placed the gut symbiont of Palomena angulosa and the gut symbiont of a cydnid stinkbug, and further outside of them were placed Escherichia coli and allied free-living $\gamma$-proteobacteria (Fig. 1).

\section{Intraspecific uniformity and exceptional diversity of gut symbiotic bacteria among pentatomid stinkbugs}

As described above, bacterial 16S rRNA gene sequences obtained from different individuals of the same species were completely or nearly identical across different populations for most of the pentatomid species examined (Fig. 1). It should be noted, however, that several exceptional cases were observed in Plautia stali, Axiagastus rosmarus and Carbula crassiventris, as reported in a previous study [22], wherein different individuals and populations of the same pentatomid species may be associated with distinct bacterial symbionts (Fig. 1).

\section{Intrageneric coherence and diversity of gut symbiotic bacteria among pentatomid stinkbugs}

In the phylogeny, the gut symbiotic bacteria of different pentatomid species belonging to the same genus tended to be closely related to each other (ex. Homalogonia spp., Menida spp., Nezara spp., Pentatoma spp., Scotinophara spp., etc.) (Fig. 1), as had been recognized in previous studies (ex. Chlorochroa spp., Eurydema spp., Edessa spp., etc.) $[13,15,20]$. On the other hand, some pentatomid species belonging to the same genus were associated with phylogenetically distinct gut symbiotic bacteria (ex. Carbula abbreviata vs. C. crassiventris, Euschistus heros vs. E. sp., Plautia stali vs. P. splendens, etc.) [13, 20-22] (Fig. 1).

\section{Multiple evolutionary origins of gut symbiotic bacteria among pentatomid stinkbugs}

These phylogenetic patterns indicate that the gut symbiotic bacteria are polyphyletic in the stinkbug family Pentatomidae, as has been suggested in previous studies [13, 20, 22, 23]. Among diverse pentatomid species, presumably, their gut symbiotic bacteria have evolved in a dynamic manner, repeatedly acquired from environmental bacteria, horizontally transferred from different stinkbug species, and/or replacing pre-existing symbiotic bacteria. For further understanding of the dynamic evolutionary processes in detail, molecular phylogenetic analysis of the host stinkbugs is also needed, which should be pursued in future studies. 


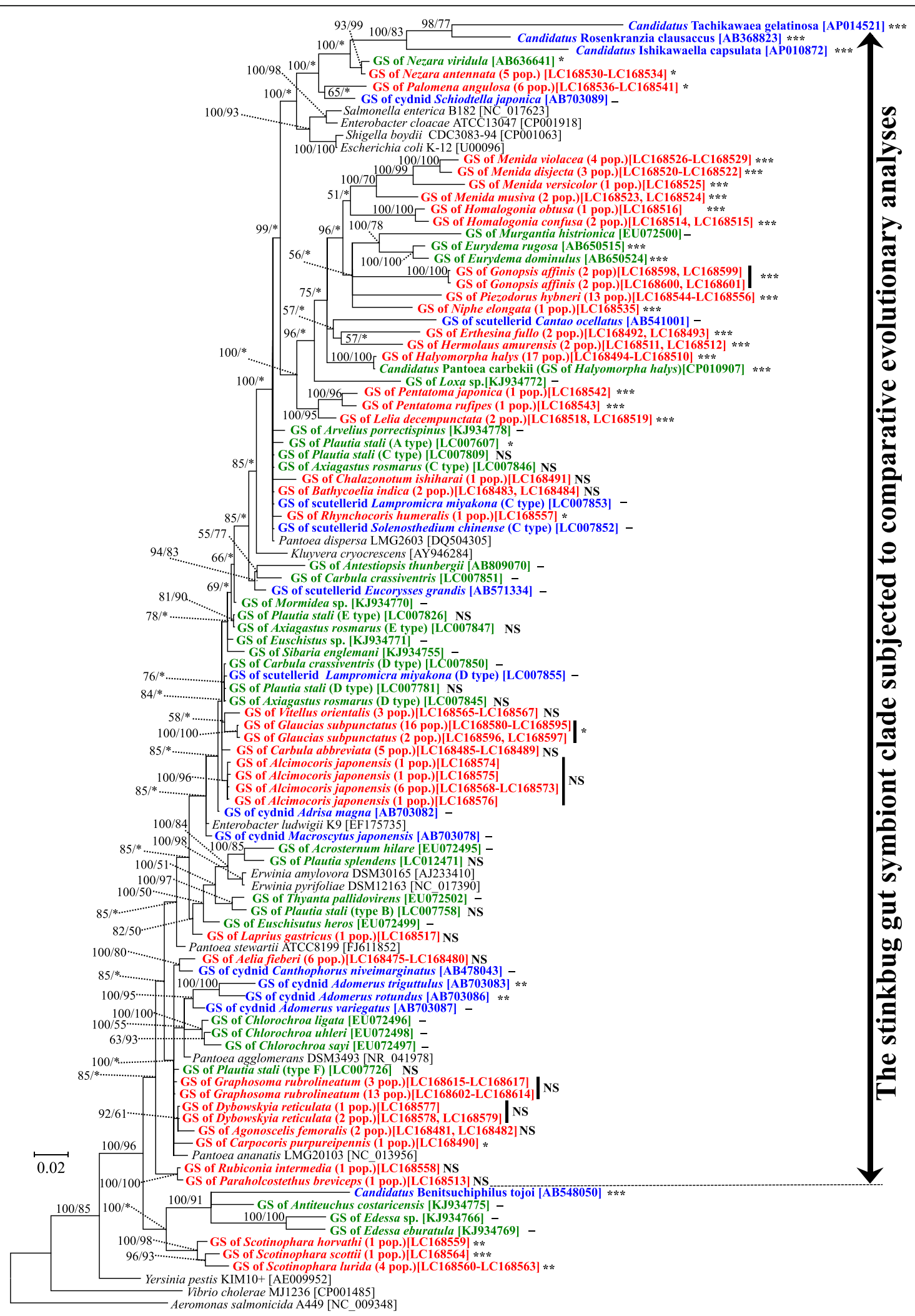

Fig. 1 (See legend on next page.) 


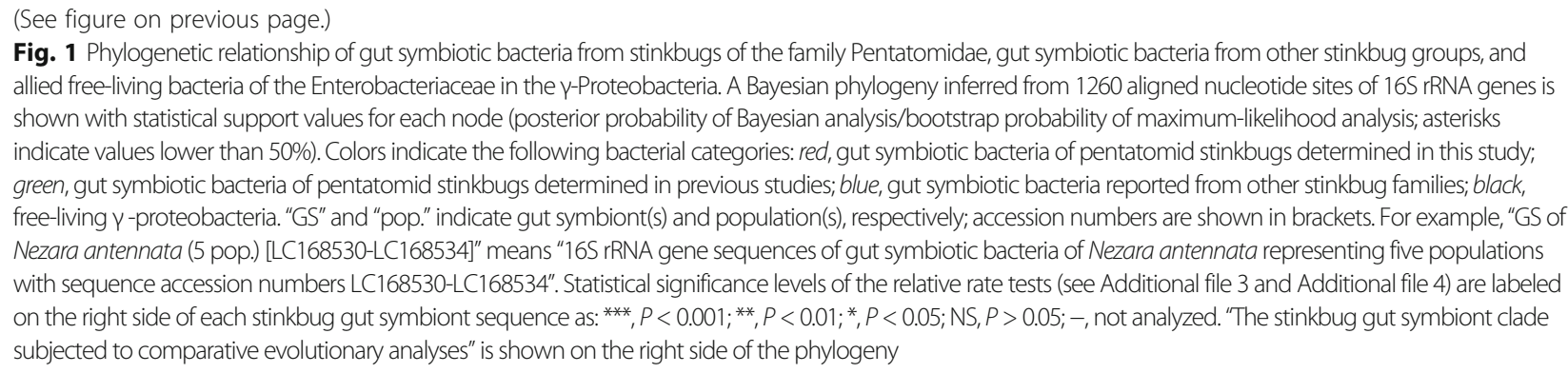

Molecular evolutionary rate and nucleotide composition among gut symbiotic bacteria of pentatomid stinkbugs In the phylogeny, the gut symbiotic bacteria conserved in their host genera (ex. those of Nezara spp., Menida spp., Homalogonia spp., Pentatoma spp., Scotinophara spp., etc.) tended to exhibit elongated branches, whereas the gut symbiotic bacteria promiscuously intermingled with free-living bacteria tended to exhibit very short branches (Fig. 1). Relative rate tests of the $16 \mathrm{~S}$ rRNA gene sequences confirmed these observations: molecular evolutionary rates were remarkably accelerated (K1/K2 values 1.5 to $2.4, P$ values $<10^{-5}$ ) in the gut symbiotic bacteria of Menida spp., Homalogonia spp., and Pentatoma spp., and other long-branched gut symbiont lineages associated with a variety of pentatomid stinkbugs including Gonopsis affinis, Piezodorus hybneri, Niphe elongata, Erthesina fullo, Hermolaus amurensis, Halyomorpha halys, and Lelia decapunctata; molecular evolutionary rates were moderately accelerated (K1/K2 values 1.1 to $1.6, P$ values 0.001-0.04) in the gut symbiotic bacteria of Scotinophara spp., Nezara spp., Palomena angulosa, Rhynchocoris humeralis, Glaucias subpunctatus and Carpocoris purpureipennis; and no accelerated molecular evolution was detected $(\mathrm{K} 1 / \mathrm{K} 2$ values around $1, P$ values $>0.05)$ in the gut symbiotic bacteria of promiscuous type from Chalazonotum ishiharai, Bathycoelia indica, Vitellus orientalis, Carbula abbreviata, Alcimocoris japonensis, Laprius gastricus, Aelia fieberi, Graphosoma rubrolineatum, Dybowskyia reticulata, Agonoscelis femoralis, Rubiconia intermedia, and Paraholcostethus breviceps (Additional file 3; Fig. 1). Notably, AT contents of the $16 \mathrm{~S}$ rRNA gene sequences exhibited overall correlation with the categories: 46.3 to $49.5 \%$ for the highly accelerated sequences; 44.3 to $45.7 \%$ for the moderately accelerated sequences; and 43.9 to $44.9 \%$ for the sequences exhibiting no acceleration (Additional file 1).

\section{Molecular evolutionary rate and nucleotide composition among gut symbiotic bacteria of other stinkbug groups and allied free-living $\gamma$-proteobacteria}

Furthermore, 16S rRNA gene sequences of gut symbiotic bacteria of stinkbugs representing the Pentatomidae and other families published in previous studies, and also those of allied free-living $\gamma$-proteobacteria, all of which are members of the Enterobacteriaceae (Fig. 1), were similarly subjected to molecular evolutionary analyses (Additional file 4). Relative rate tests revealed the following patterns: in the genome-reduced and co-speciating gut symbiotic bacteria from the stinkbug families Plataspidae, Acanthosomatidae and Urostylididae [11, 12, 18], molecular evolutionary rates were extremely accelerated (K1/K2 values 3.4-5.0, $P$ values $<10^{-7}$ ) with extremely high AT contents $(>50.3 \%)$; in the genome-reduced gut symbiotic bacterium from the stinkbug family Parastrachiidae [37], a highly accelerated molecular evolutionary rate was observed $\left(\mathrm{K} 1 / \mathrm{K} 2\right.$ value $1.5, P$ value $\left.<10^{-4}\right)$ with extremely high AT content (51.0\%); in the uncultivable gut symbiotic bacteria of pentatomid stinkbugs, some species (Eurydema spp. and Halyomorpha halys) $[15,28]$ exhibited remarkably high molecular evolutionary rates (K1/K2 values $1.8-2.0, P$ values $<10^{-7}$ ) with relatively high AT contents (48.1-48.6\%), other species (Nezara viridula and Plautia stali type A) [14, 22] showed moderately high molecular evolutionary rates (K1/K2 values $1.1-1.6$, $P$ values around 0.02 ) with low AT contents (44.7-45.7\%), and other species (Plautia splendens and Plautia stali B type) $[21,22]$ entailed no accelerated molecular evolution $(\mathrm{K} 1 / \mathrm{K} 2$ values around $1.1, P$ values $>0.05)$ with low AT contents (43.9-44.3\%); in the cultivable gut symbiotic bacteria of pentatomid stinkbugs (Axiagastus rosmarus C-E types and Plautia stali C-F types) [22], no accelerated molecular evolution was detected (K1/K2 values around $1.0, P$ values $0.3-1.0$ ) with low AT contents (44.4-44.9\%).

\section{Relationships between molecular evolutionary rate, nucleotide composition, genome size, and cultivability of gut symbiotic bacteria of pentatomid stinkbugs}

Previous studies have revealed that, through intimate and long-lasting host-symbiont co-evolution, obligate endocellular symbiotic bacteria of diverse insects tend to exhibit a characteristic genomic syndrome, including accelerated molecular evolution, AT-biased nucleotide composition, massive gene loss, and reduced genome size. This has been ascribed to stable and nutrition-rich endocellular environment and to strong population bottlenecks associated with the lifestyles of vertically-transmitted 
symbiotic bacteria [53-56]. Recently, remarkable genome reduction has also been identified among extracellular gut symbiotic bacteria of various stinkbugs $[11,12,17,18,22$, $28,37,41]$, elucidating that endocellularity cannot be the main driver of the symbiosis-associated reductive genome evolution $[7,11,57]$. In this study, a wide variety of gut symbiotic bacteria of pentatomid stinkbugs at different evolutionary stages, ranging from cultivable through uncultivable to genome-reduced, were found together with free-living bacteria and diverse gut symbiotic bacteria of other stinkbug families (Fig. 1), which provided an ideal opportunity to comparatively analyze the evolutionary processes underpinning the gut symbiotic associations. For that purpose, we focused on "the stinkbug gut symbiont clade subjected to comparative evolutionary analyses" (see Fig. 1 on the right side), defined an outgroup of the clade (Yersinia pestis KIM10+), and calculated the value $\mathrm{K}$ (genetic distance from the outgroup) for each member of the clade (Additional file 5), which enabled comparison of molecular evolutionary rates across all members of the clade. Figure 2a shows the relationship between $\mathrm{K}$ values and AT contents within the clade, indicating a fairly strong positive correlation $(\mathrm{R}=0.955)$. On the plot, the gut symbionts of diverse pentatomid stinkbugs (black, red and blue) were scattered from the lower left (= low evolutionary rate and low AT content) to the upper right (= high evolutionary rate and high AT content). When uncultivable and genome-reduced gut symbionts of other stinkbug families (green) and free-living $\gamma$-proteobacteria (grey) were analyzed on the same plot, the former tended to be located on the upper right extreme (= highest evolutionary rate and highest AT content), whereas the latter were concentrated on the lower left extreme (= lowest evolutionary rate and lowest AT content). For some of the gut symbionts of pentatomid stinkbugs, we examined whether they are cultivable or not $[14,15,21,22,28]$ (see Additional file 2). Interestingly, the uncultivable gut symbionts of pentatomid stinkbugs (red) tended to be distributed from the middle to the lower left, whereas the cultivable gut symbionts of pentatomid stinkbugs (blue) were concentrated on the lower left extreme as the free-living $\gamma$-proteobacteria. Figure $2 \mathrm{~b}$ shows the relationship between $\mathrm{K}$ values and genome sizes within the clade, exhibiting a strong negative correlation $(R=-0.847)$. In the plot, the uncultivable and genomereduced gut symbionts of other stinkbug families (green) were located to the lower right extreme, the cultivable gut symbionts of pentatomid stinkbugs (blue) and the free-living $\gamma$-proteobacteria (grey) were concentrated to the upper left extreme, and the uncultivable gut symbionts of pentatomid stinkbugs (red) exhibited an intermediate distribution. Figure 2c shows the relationship between AT contents and $K$ values within the clade, exhibiting a similar pattern to Fig. $2 \mathrm{~b}$ with a strong negative correlation $(R=-0.778)$.

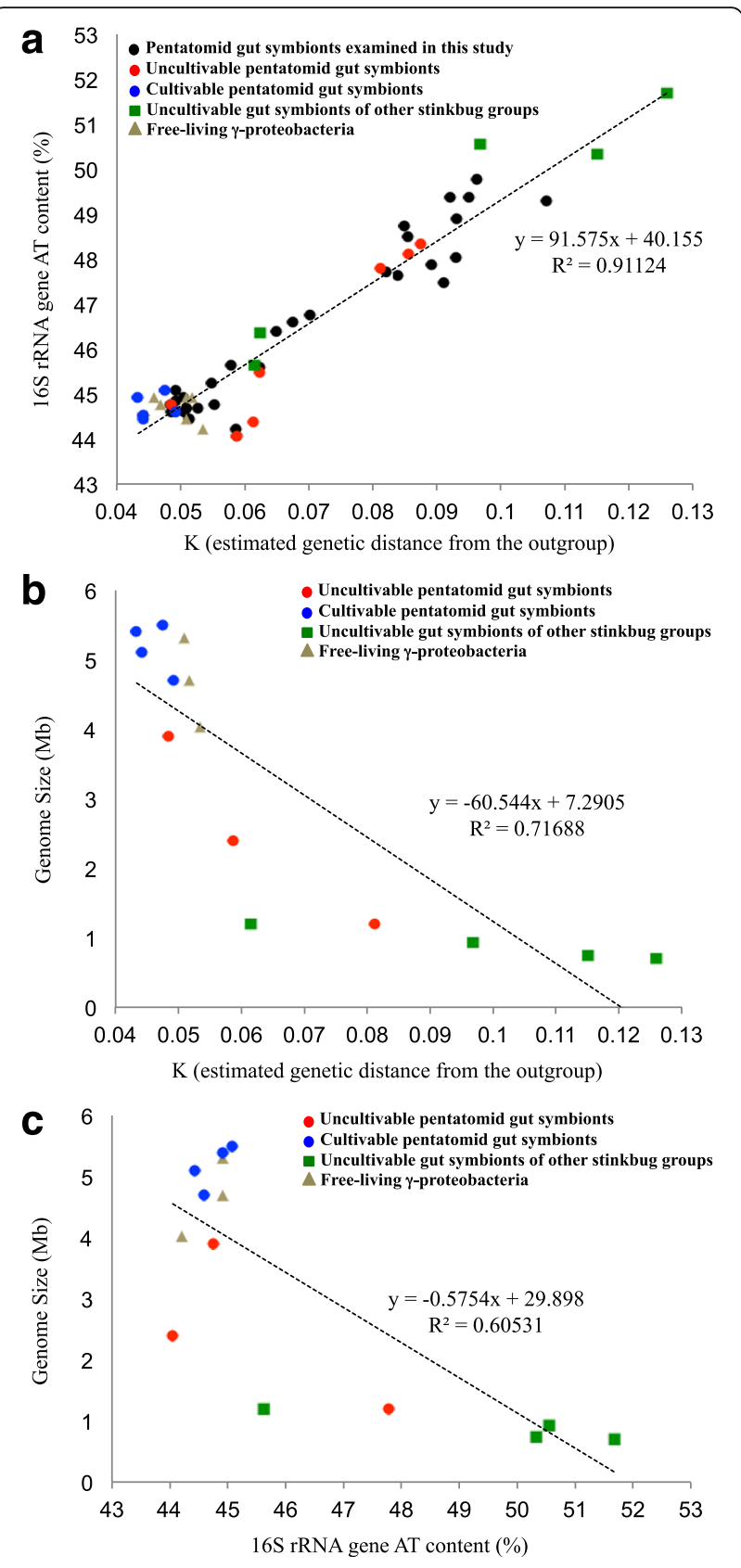

Fig. 2 Relationships between molecular evolutionary rate, nucleotide composition, and genome size between gut symbiotic bacteria of pentatomid stinkbugs, gut symbiotic bacteria of other stinkbug groups, and allied free-living $\mathrm{Y}$-proteobacteria. a K vs. AT content of $16 \mathrm{~S}$ rRNA gene. b K vs. genome size. c AT content of $16 \mathrm{~S}$ rRNA gene vs. genome size. $\mathrm{K}$ is defined as estimated genetic distance from the outgroup of "the stinkbug gut symbiont clade subjected to comparative evolutionary analyses" (see Fig. 1 and Additional file 5), reflecting the molecular evolutionary rate of the lineage

Multiple evolutionary origins and different evolutionary stages of gut symbiotic bacteria in the Pentatomidae These results collectively highlight the following dynamic evolutionary aspects of the gut symbiotic bacteria in the 
stinkbug family Pentatomidae: (i) each of the pentatomid host species examined in this study is monosymbiotically associated with a specific $\gamma$-proteobacterium within the midgut symbiotic organ; (ii) gut symbiotic bacteria are of multiple evolutionary origins in the Pentatomidae, presumably originating from free-living $\gamma$-proteobacteria belonging to the Enterobacteriaceae repeatedly; (iii) some are of relatively old origin and stably maintained across closely related host species, some are of relatively recent origin and stably associated with a specific host species, and others are of promiscuous nature whose infections may be polymorphic within and/or between host species; (iv) stable gut symbiotic bacteria tend to exhibit uncultivability, accelerated molecular evolution, AT-biased nucleotide composition and reduced genome; (v) by contrast, promiscuous gut symbiotic bacteria tend to exhibit cultivability, non-accelerated molecular evolution, unbiased nucleotide composition and nonreduced genome, which are similar to free-living environmental bacteria; and (vi) a number of gut symbiont lineages at different evolutionary stages of different symbiotic intimacy coexist within the stinkbug family Pentatomidae.

\section{Conclusions}

In conclusion, gut symbiotic bacteria have evolved repeatedly and dynamically within the stinkbug family Pentatomidae, which have plausibly entailed frequent acquisitions, losses, replacements, and transfers, and sometimes led to establishment of relatively stable host-symbiont associations. The remarkable diversity of host-symbiont relationships in the Pentatomidae provides an ideal arena for investigating the evolution of symbiosis not only experimentally but also theoretically. The mechanisms and processes underlying stability vs. promiscuity, vertical transmission vs. horizontal acquisition, cultivability vs. uncultivability, etc. among the gut symbiotic bacteria are of particular interest. For example, the stability of host-symbiont associations must be relevant to stable vertical transmission ensured by maternal deposition of symbiont-containing excretion onto the egg surface [21], and to selection of specific bacteria mediated by a symbiont sorting organ in the stinkbug midgut [58]. What ecological factors are relevant to the symbiont transmission mode (vertical, horizontal, or environmental) is an important theoretical issue [59]. It is unknown what biological functions underpin the beneficial roles of the diverse symbiotic bacteria for the host stinkbugs, to which genomic and physiological comparisons among the symbiotic bacteria may provide valuable insights [22, 57]. A number of pentatomid stinkbugs are notorious as agricultural pests [60], and biological understanding of their symbiotic bacteria may also contribute to their control and management. In this study, we describe the gut symbiotic bacteria of 35 Japanese pentatomid species, which account for over $40 \%$ of all pentatomid species reported from Japan [61]. In the world, some 5000 pentatomid stinkbugs have been described [62], and we suggest that a survey of their symbiotic microbial associates will lead to further discoveries of general relevance.

\section{Additional files}

Additional file 1: Stinkbug samples used in this study. (XLSX $18 \mathrm{~kb}$ )

Additional file 2: Bacterial 16S rRNA gene sequences of gut symbionts of stinkbugs and free-living bacteria retrieved from the DNA databases and analyzed in this study. (XLSX $18 \mathrm{~kb}$ )

Additional file 3: Relative rate tests of 16S rRNA gene sequences of the gut symbionts of pentatomid stinkbugs in comparison with allied free-living bacteria. (DOCX 163 kb)

Additional file 4: Relative rate tests of 165 rRNA gene sequences of the cultivable and uncultivable stinkbug gut symbionts in comparison with allied free-living bacteria. (DOCX $152 \mathrm{~kb}$ )

Additional file 5: Bacterial taxa and their relevant parameters analyzed in Fig. 2. (DOCX $136 \mathrm{~kb})$

\section{Acknowledgments}

We thank M. Baba, Y. G. Baba, E. Hara, D. Haraguchi, C. Himuro, H. Hirayama, S. Kada, N. Kaiwa, T. Kawagoe, S. Koike, S. Kudo, Y. Osada, M. Sakakibara, K. O. Shiromoto, K. Takahashi, H. Toju, N. Tsurusaki, H. Watanabe for providing insect samples, and N. Nikoh for comments on the manuscript.

\section{Funding}

This study was supported by JSPS Kakenhi Grant Numbers JP25221107 to TF and JP15K21209 to TH.

\section{Availability of data and materials}

The nucleotide sequences determined in this study were deposited in the DNA Data Bank of Japan (http://www.ddbj.nig.ac.jp/index-e.html) under accession numbers LC168475-LC168617.

\section{Authors' contributions}

$\mathrm{TH}$ and YM primarily and equally contributed to the work. TH, YM and YK collected the insects and prepared DNA samples. TH mainly performed PCR, cloning and DNA sequencing. YM mainly conducted molecular phylogenetic and evolutionary analyses. TF, TH and YM wrote the manuscript. All authors read and approved the final manuscript.

\section{Competing interests}

The authors declare that they have no competing interests.

\section{Consent for publication}

Not applicable.

\section{Ethics approval and consent to participate} Not applicable.

\section{Author details}

${ }^{1}$ National Institute of Advanced Industrial Science and Technology (AIST), Tsukuba 305-8566, Japan. 'Faculty of Science, Kyushu University, Fukuoka 819-0395, Japan. ${ }^{3}$ Tropical Biosphere Research Center, University of the Ryukyus, Okinawa 903-0213, Japan. ${ }^{4}$ National Institute of Advanced Industrial Science and Technology (AIST), Hokkaido Center, Sapporo 062-8517, Japan. ${ }^{5}$ Department of Biological Sciences, Graduate School of Science, University of Tokyo, Tokyo 113-0033, Japan. ${ }^{6}$ Graduate School of Life and Environmental Sciences, University of Tsukuba, Tsukuba 305-8572, Japan. 


\section{Received: 23 September 2016 Accepted: 26 November 2016}

Published online: 30 November 2016

\section{References}

1. Buchner P. Endosymbiosis of animals with plant microorganisms. New York: Interscience; 1965

2. Bourtzis K, Miller TA. Insect symbiosis. Boca Raton: CRC Press; 2003.

3. Zchori-Fein E, Bourtzis K. Manipulative tenants: bacteria associated with arthropods. Boca Raton: CRC Press; 2011.

4. Glasgow H. The gastric caeca and the caecal bacteria of the Heteroptera. Biol Bull. 1914:3:101-71.

5. Rosenkranz W. Die Symbiose der Pentatomiden. Z Morphol Okol Tiere. 1939; 36:279-309.

6. Kikuchi Y, Hosokawa T, Fukatsu T. Diversity of bacterial symbiosis in stinkbugs. In: Dijk TV, editor. Microbial ecology research trends. New York: Nova Science Publishers, Inc; 2008. p. 39-63.

7. Salem H, Florez L, Gerardo N, Kaltenpoth M. An out-of-body experience: the extracellular dimension for the transmission of mutualistic bacteria in insects. Proc R Soc B. 2015;282:20142957.

8. Abe Y, Mishiro K, Takanashi M. Symbiont of brown-winged green bug, Plautia stali Scott. Jpn J Appl Entomol Zool. 1995;39:109-15.

9. Fukatsu T, Hosokawa T. Capsule-transmitted gut symbiotic bacterium of the Japanese common plataspid stinkbug, Megacopta punctatissima. Appl Environ Microbiol. 2002;68:389-96.

10. Prado SS, Rubinoff D, Almeida RPP. Vertical transmission of a pentatomid caeca-associated symbiont. Ann Entomol Soc Am. 2006;99:577-85.

11. Hosokawa T, Kikuchi Y, Nikoh N, Shimada M, Fukatsu T. Strict host-symbiont cospeciation and reductive genome evolution in insect gut bacteria. PLoS Biol. 2006:4:e337.

12. Kikuchi Y, Hosokawa T, Nikoh N, Meng XY, Kamagata Y, Fukatsu T. Hostsymbiont co-speciation and reductive genome evolution in gut symbiotic bacteria of acanthosomatid stinkbugs. BMC Biol. 2009;7:2.

13. Prado SS, Almeida RPP. Phylogenetic placement of pentatomid stink bug gut symbionts. Curr Microbiol. 2009;58:64-9.

14. Tada A, Kikuchi Y, Hosokawa T, Musolin DL, Fujisaki K, Fukatsu T. Obligate association with gut bacterial symbiont in Japanese populations of southern green stinkbug Nezara viridula (Heteroptera: Pentatomidae). Appl Entomol Zool. 2011;46:483-8.

15. Kikuchi Y, Hosokawa T, Nikoh N, Fukatsu T. Gut symbiotic bacteria in the cabbage bugs Eurydema rugosa and Eurydema dominulus (Heteroptera: Pentatomidae). Appl Entomol Zool. 2012;47:1-8.

16. Hosokawa T, Hironaka M, Mukai H, Inadomi K, Suzuki N, Fukatsu T. Mothers never miss the moment: a fine-tuned mechanism for vertical symbiont transmission in a subsocial insect. Anim Behav. 2010;83:293-300.

17. Hosokawa T, Hironaka M, Inadomi K, Mukai H, Nikoh N, Fukatsu T. Diverse strategies for vertical symbiont transmission among subsocial stinkbugs. PLoS One. 2013;8:e65081.

18. Kaiwa N, Hosokawa T, Nikoh N, Tanahashi M, Moriyama M, Meng XY, Maeda T, Yamaguchi K, Shigenobu S, Ito M, Fukatsu T. Symbiont-supplemented maternal investment underpinning host's ecological adaptation. Curr Biol. 2014:24:2465-70

19. Taylor CM, Coffey PL, DeLay BD, Dively GP. The importance of gut symbionts in the development of the brown marmorated stink bug Halyomorpha halys (Stål). PLoS One. 2014;9:e90312.

20. Bistolas KSI, Sakamoto RI, Fernandes JAM, Goffredi SK. Symbiont polyphyly, co-evolution, and necessity in pentatomid stinkbugs from Costa Rica. Front Microbiol. 2015:5:349.

21. Hayashi T, Hosokawa T, Meng XY, Koga R, Fukatsu T. Female-specific specialization of a posterior end region of the midgut symbiotic organ in Plautia splendens and allied stinkbugs. Appl Environ Microbiol. 2015;81:2603-11.

22. Hosokawa T, Ishii Y, Nikoh N, Fujie M, Satoh N, Fukatsu T. Obligate bacterial mutualists evolving from environmental bacteria in natural insect populations. Nat Microbiol. 2016;1:15011.

23. Duron $\mathrm{O}$, Noël V. A wide diversity of Pantoea lineages are engaged in mutualistic symbiosis and cospeciation processes with stinkbugs. Environ Microbiol Rep. 2016. doi:10.1111/1758-2229.12432.

24. Karamipour N, Mehrabadi M, Fathipour Y. Gammaproteobacteria as essential primary symbionts in the striped shield bug, Graphosoma lineatum (Hemiptera: Pentatomidae). Sci Rep. 2016;6:33168.

25. Hosokawa T, Kikuchi Y, Nikoh N, Fukatsu T. Polyphyly of gut symbionts in stinkbugs of the family Cydnidae. Appl Environ Microbiol. 2012;78:4758-61.
26. Matsuura Y, Hosokawa T, Serracin M, Tulgetske GM, Miller TA, Fukatsu T. Bacterial symbionts of a devastating coffee plant pest, the stinkbug Antestiopsis thunbergii (Hemiptera: Pentatomidae). Appl Environ Microbiol. 2014:80:3769-75.

27. Bansal R, Michel AP, Sabree ZL. The crypt-dwelling primary bacterial symbiont of the polyphagous pentatomid pest Halyomorpha halys (Hemiptera: Pentatomidae). Environ Microbiol. 2014;43:617-25.

28. Kenyon LJ, Meulia T, Sabree ZL. Habitat visualization and genomic analysis of "Candidatus Pantoea carbekii", the primary symbiont of the brown marmorated stink bug. Genome Biol Evol. 2015;7:620-35.

29. Fukatsu T. Acetone preservation: a practical technique for molecular analysis. Mol Ecol. 1999;8:1935-45.

30. Fukatsu T, Nikoh N. Two intracellular symbiotic bacteria of the mulberry psyllid Anomoneura mori (Insecta, Homoptera). Appl Environ Microbiol. 1998:64:3599-606.

31. Katoh K, Standley DM. MAFFT multiple sequence alignment software version 7: improvements in performance and usability. Mol Biol Evol. 2013; 30:772-80.

32. Darriba D, Taboada GL, Doallo R, Posada D. jModelTest 2: more models, new heuristics and parallel computing. Nat Methods. 2012;9:772.

33. Guindon S, Gascuel O. A simple, fast, and accurate algorithm to estimate large phylogenies by maximum likelihood. Syst Biol. 2003;52:696-704.

34. Ronquist F, Teslenko M, van der Mark P, Ayres DL, Darling A, Höhna S, Larget B, Liu L, Suchard MA, Huelsenbeck JP. MrBayes 3.2: efficient Bayesian phylogenetic inference and model choice across a large model space. Syst Biol. 2012;61:539-42.

35. Stamatakis A. RAxML version 8: a tool for phylogenetic analysis and postanalysis of large phylogenies. Bioinformatics. 2014;30:1312-3.

36. Robinson-Rechavi M, Huchon D. RRTree: relative-rate tests between groups of sequences on a phylogenetic tree. Bioinformatics. 2000;16:296-7.

37. Hosokawa T, Kikuchi Y, Nikoh N, Meng XY, Hironaka M, Fukatsu T. Phylogenetic position and peculiar genetic traits of a midgut bacterial symbiont of the stinkbug Parastrachia japonensis. Appl Environ Microbiol. 2010:76:4130-5.

38. Kaiwa N, Hosokawa T, Kikuchi Y, Nikoh N, Meng XY, Kimura N, Ito M, Fukatsu T. Primary gut symbiont and secondary, Sodalis-allied symbiont in the scutellerid stinkbug Cantao ocellatus. Appl Environ Microbiol. 2010;76:3486-94.

39. Kaiwa N, Hosokawa T, Kikuchi Y, Nikoh N, Meng XY, Kimura N, Ito M, Fukatsu T. Bacterial symbionts of the giant jewel stinkbug Eucoryssus grandis (Hemiptera: Scutelleridae). Zool Sci. 2011;28:169-74.

40. Baba N, Hironaka M, Hosokawa T, Mukai H, Nomakuchi S, Ueno T. Trophic eggs compensate for poor offspring feeding capacity in a subsocial burrower bug. Biol Let. 2011;7:194-6.

41. Nikoh N, Hosokawa T, Oshima K, Hattori M, Fukatsu T. Reductive evolution of bacterial genome in insect gut environment. Genome Biol Evol. 2011;3:702-14.

42. Blattner FR, Plunkett G, Bloch CA, Perna NT, Burland V, Riley M, ColladoVides J, Glasner JD, Rode CK, Mayhew GF, Gregor J, Davis NW, Kirkpatrick HA, Goeden MA, Rose DJ, Mau B, Shao Y. The complete genome sequence of Escherichia coli K-12. Science. 1997;277:1453-62.

43. Spröer C, Mendrock U, Swiderski J, Lang E, Stackebrandt E. The phylogenetic position of Serratia, Buttiauxella and some other genera of the family Enterobacteriaceae. Int J Syst Evol Microbiol. 1999;49:1433-8.

44. Deng W, Burland V, Plunkett G, Boutin A, Mayhew GF, Liss P, Perna NT, Rose DJ, Mau B, Zhou S, Schwartz DC, Fetherston JD, Lindler LE, Brubaker RR, Plano GV, Straley SC, McDonough KA, Nilles ML, Matson JS, Blattner FR, Perry RD. Genome sequence of Yersinia pestis KIM. J Bacteriol. 2002;184:4601-11.

45. Li H, Medina F, Vinson SB, Coates CJ. Isolation, characterization, and molecular identification of bacteria from the red imported fire ant (Solenopsis invicta) midgut. J Invertebr Pathol. 2005;89:203-9.

46. Reith ME, Singh RK, Curtis B, Boyd JM, Bouevitch A, Kimball J, Munholland J, Murphy C, Sarty D, Williams J, Nash JHE, Johnson SC, Brown LL. The genome of Aeromonas salmonicida subsp. salmonicida A449: insights into the evolution of a fish pathogen. BMC Genomics. 2008:9:427.

47. Rezzonico F, Smits THM, Montesinos E, Frey JH, Duffy B. Genotypic comparison of Pantoea agglomerans plant and clinical strains. BMC Microbiol. 2009:9:204.

48. Ren Y, Ren Y, Zhou Z, Guo X, Li Y, Feng L, Wang L. Complete genome sequence of Enterobacter cloacae subsp. cloacae type strain ATCC 13047. J Bacteriol. 2010;192:2463-4.

49. Smits THM, Jaenicke S, Rezzonico F, Kamber T, Goesmann A, Frey JE, Duffy B. Complete genome sequence of the fire blight pathogen Erwinia pyrifoliae 
DSM $12163^{\top}$ and comparative genomic insights into plant pathogenicity. BMC Genomics. 2010;11:2.

50. De Maayer P, Chan WY, Venter SN, Toth IK, Birch PRJ, Joubert F, Coutinho TA. Genome sequence of Pantoea ananatis LMG20103, the causative agent of eucalyptus blight and dieback. J Bacteriol. 2010;192:2936-7.

51. Bars HL, Bousarghin L, Bonnaure-Mallet M, Jolivet-Gougeon A, Barloy-Hubler F. Complete genome sequence of the strong mutator Salmonella enterica subsp. enterica serotype Heidelberg strain B182. J Bacteriol. 2012;194:3537-8.

52. Kania DA, Hazen TH, Hossain A, Nataro JP, Rasko DA. Genome diversity of Shigella boydii. Pathog Dis. 2016;74:ftw027.

53. Wernegreen JJ. Genome evolution in bacterial endosymbionts of insects. Nat Rev Genet. 2002;3:850-61.

54. Moran NA, McCutcheon JP, Nakabachi A. Genomics and evolution of heritable bacterial symbionts. Annu Rev Genet. 2008;42:165-90.

55. Moya A, Peretó J, Gil R, Latorre A. Learning how to live together: genomic insights into prokaryote-animal symbioses. Nat Rev Genet. 2008;9:218-29.

56. McCutcheon JP, Moran NA. Extreme genome reduction in symbiotic bacteria. Nat Rev Microbiol. 2012;10:13-26.

57. Otero-Bravo A, Sabree ZL. Inside or out? Possible genomic consequences of extracellular transmission of crypt-dwelling stinkbug mutualists. Front Ecol Evol. 2015;3:64.

58. Ohbayashi T, Takeshita K, Kitagawa W, Nikoh N, Koga R, Meng XY, Tago K, Hori T, Hayatsu M, Asano K, Kamagata Y, Lee BL, Fukatsu T, Kikuchi Y. Insect's intestinal organ for symbiont sorting. Proc Natl Acad Sci U S A. 2015;112:E5179-88.

59. Bright M, Bulgheresi S. A complex journey: transmission of microbial symbionts. Nat Rev Microbiol. 2010;8:218-30.

60. Schaefer CW, Panizzi AR. Heteroptera of economic importance. Boca Raton: CRC Press; 2000

61. Ishikawa T, Takai M, Yasunaga T. A field guide to Japanese bugs III. Tokyo: Zenkoku Noson Kyoiku Kyokai; 2012.

62. Aukema B, Rieger C. Catalogue of the Heteroptera of the Palaearctic Region. Amsterdam: The Netherlands Entomological Society; 2006.

\section{Submit your next manuscript to BioMed Central and we will help you at every step:}

- We accept pre-submission inquiries

- Our selector tool helps you to find the most relevant journal

- We provide round the clock customer support

- Convenient online submission

- Thorough peer review

- Inclusion in PubMed and all major indexing services

- Maximum visibility for your research

Submit your manuscript at www.biomedcentral.com/submit

C) Biomed Central 